Nota científica

(Short communication)

\title{
REGISTROS DE THRAUPIS EPISCOPUS (LINNAEUS, 1766) EN LA CIUDAD DE CHILPANCINGO, GUERRERO, MÉXICO
}

\section{RECORDS OF THRAUPIS EPISCOPUS (LINNAEUS, 1766) IN CHILPANCINGO CITY, STATE OF GUERRERO, MEXICO}

\author{
MARISOl CASTRO TORREBLANCA ${ }^{12 *}$ Y EPIFANIO BLANCAS CALVA ${ }^{3}$
}

\author{
${ }^{1}$ Posgrado en Ciencias Biológicas, Universidad Nacional Autónoma de México. Apartado postal 70-153, CP \\ 04510 Ciudad de México, México.<balam_mampar@hotmail.com> \\ ${ }^{2}$ Universidad Nacional Autónoma de México, Facultad de Estudios Superiores Zaragoza, UMIEZ. Av. Batalla del \\ 5 de mayo s/n Col. Ejército de Oriente, Iztapalapa. Apartado Postal 70-153,04510, CP 09230, Ciudad de México. \\ ${ }^{3}$ Instituto de Investigación Científica Área de Ciencias Naturales, Universidad Autónoma de Guerrero. Av. Lázaro \\ Cárdenas s/n, interior del Jardín Botánico, Ciudad Universitaria, Chilpancingo, Guerrero, CP 39087, México. \\ <ebcalva@yahoo.com.mx> \\ *Autor para correspondencia: <balam_mampar@hotmail.com> \\ Recibido: 24/05/2017; aceptado: 07/12/2017; publicado en línea: 16/03/2018 \\ Editor responsable: Ricardo Rodríguez Estrella
}

\begin{abstract}
Castro-Torreblanca, M. \& Blancas-Calva, E. (2018) Registros de Thraupis episcopus (Linnaeus, 1766) en la ciudad de Chilpancingo, Guerrero, México. Acta Zoológica Mexicana (n.s), 34(1), 1-3
\end{abstract}

RESUMEN. Se registró la presencia de dos individuos de la tángara azul-gris (Thraupis episcopus) en un área urbana de la ciudad de Chilpancingo, estado de Guerrero. Estos registros son importantes porque aumenta en $250 \mathrm{~km}$ el límite suroeste conocido de distribución de la especie.

La tángara azul-gris (Thraupis episcopus), es una especie de traúpido neotropical, residente y común. Se distribuye desde el noreste de México hasta el centro de Brasil, norte de Bolivia y este de Perú (Clements, 1991; Dickinson, 2003), entre los 0 y los 1500 msnm (AOS, 1998). En México, su distribución incluye los estados del sur y sureste; San Luis Potosí, Hidalgo, Puebla, Veracruz, Tabasco, Campeche, Quintana Roo, Chiapas y Oaxaca (Howell \& Webb, 1995), recientemente se ha registrado en la península de Yucatán (Navarro et al., 2003), Nuevo León (Monroy \& Grosselet, 2008) y Tamaulipas (Rodríguez-Ruiz et al., 2011). Se ha documentado que tiene preferencia por hábitats modificados antropogénicamente; áreas abiertas y semiabiertas, plantaciones, jardines en las afueras de las
Castro-Torreblanca, M. \& Blancas-Calva, E. (2018) Records of Thraupis episcopus (Linnaeus, 1766) in Chilpancingo city, State of Guerrero, Mexico. Acta Zoológica Mexicana (n.s), 34(1), 1-3

ABSTRACT. We recorded the presence of two individuals of the Blue-gray Tanager (Thraupis episcopus) on an urban area in Chilpancingo city in the state of Guerrero. These records are important because increases in $250 \mathrm{~km}$ the known southwest limit of the distribution of the species.

ciudades, parques, plazas, bordes de bosques húmedos y bosques secundarios (Peterson \& Chalif, 1989; Howell \& Webb, 1995; AOS, 1998; Rodríguez-Ruiz et al., 2011). La distribución geográfica histórica no abarca el estado de Guerrero (Peterson \& Chalif, 1989; Howell \& Webb, 1995; Navarro-Sigüenza \& Peterson, 2007) y la literatura actual no la incluye en el listado de las especies de aves para el mismo (Navarro-Sigüenza, 1998; Navarro et al., 2016).

El 11 de enero de 2017 a las 07:35 horas la primera autora observó ocasionalmente a un individuo de tángara-azul gris vocalizando y perchando por unos segundos en un árbol de mamey (Pouteria sapota) en un patio de una vivienda particular ubicado al noroeste de la ciudad 
de Chilpancingo, Guerrero (17 33' 21.16" N, 99 31' 26.31" O; $1445 \mathrm{msnm}$ ). Asimismo, el 22 de abril de 2017 a las 19:22 horas observamos a dos individuos de tángara azul-gris vocalizando y forrajeando en árboles de lluvia de oro (Cassia fistula) plantados en un andador central de la misma ciudad (17\% 33' 11.51" N, 99 30' 09.07" $\mathrm{O} ; 1267 \mathrm{msnm}$ ). Posteriormente, el 11 de junio de 2017 a las 18:56 horas observamos a dos individuos forrajeando en árboles de tulipán africano (Spathodea campanulata; Fig. 1), en el zócalo de la ciudad ( $17^{\circ} 33^{\prime} 07.84^{\prime \prime} \mathrm{N}, 99^{\circ}$ 30 ' 04.87" O; $1268 \mathrm{msnm}$ ). El forrajeo consistió en buscar pequeños artrópodos sobre la corteza de las ramas de los árboles, después de un par de minutos un individuo voló hacia un árbol de tabachín (Delonix regia) ubicado en el costado este de la catedral de Chilpancingo, donde comenzó a vocalizar y a emitir llamados con lo que el segundo individuo de tángara azul-gris voló hacia él para acompañarlo. Estos especímenes fueron fotografiados con una cámara Nikon ${ }^{\circledR}$ D5100, lente Nikon ${ }^{\circledR}$ de 50-300 mm. En visitas posteriores al parque central de la ciudad, en los meses de junio y julio de 2017 hemos observado a dos individuos de T. episcopus en diferentes horarios.

Para la determinación taxonómica de los individuos observados utilizamos las siguientes señas de campo: la coloración gris-azulado pálido en el dorso, con azul grisáceo brillante en las alas y la cola (Peterson \& Chalif, 1989), así como su voz característica ruidosa, compuesta por vocalizaciones suaves mezcladas con silbidos agudos; tssiuu-tssiuu seguido de notas cortas y repetitivas; siendo esta voz coherente con sonidos, cantos y llamados de T. episcopus disponibles en Macaulay Library (2017) y Xeno-canto (2017).

El límite de distribución en la vertiente del Pacífico de esta especie, se cita para el este y sureste del estado de Oaxaca (eBird, 2017; Rodríguez-Ruiz et al., 2011; Navarro et al., 2016). Asimismo, existen dos registros aislados en las costas del estado de Guerrero, uno logrado en Acapulco (16 51' 16.1” N, 99 53' 49.0" O, 3 msnm) el 6 de junio de 2006, y otro en Ixtapa, Zihuatanejo (17 $39^{\circ}$ $50.6^{\prime \prime} \mathrm{N}, 101^{\circ} 36^{\prime} 19.8^{\prime \prime} \mathrm{O}, 10 \mathrm{msnm}$ ) el 23 de febrero de 2014 (eBird, 2017). También existe un registro realizado el 13 de noviembre de 2016 en Jardines de México en Tehuixtla, Morelos (18 36' 30.41” N, 99 17' 31.97” O; $961 \mathrm{msnm}$; eBird, 2017). Recientemente, Blancas-Calva et al. (2017) registraron la presencia de T. episcopus en las localidades de San Luis Acatlán y Jolotichán, en la región sureste del estado de Guerrero, en ambientes urbanos.

Las observaciones que reportamos para la ciudad de Chilpancingo, son relevantes porque desplazan el límite de distribución de T. episcopus aproximadamente $250 \mathrm{~km}$ del área de su distribución reconocida en la vertiente del Pacífico (Navarro et al., 2016), y $90 \mathrm{~km}$ de la costa del estado de Guerrero (eBird, 2017). La presencia de la tángara azul-gris puede deberse a individuos escapados de cautiverio. Sin embargo, no hemos observado en cautiverio ni a la venta ejemplares de esta especie en el municipio y/o alrededores. Por otra parte, en los últimos diez años se ha registrado una ampliación del área de distribución de esta especie, tanto en el noreste del país (Monroy \& Grosselet, 2008; Rodríguez-Ruiz et al., 2011), como en la península de Yucatán, este del estado de Oaxaca (eBird,
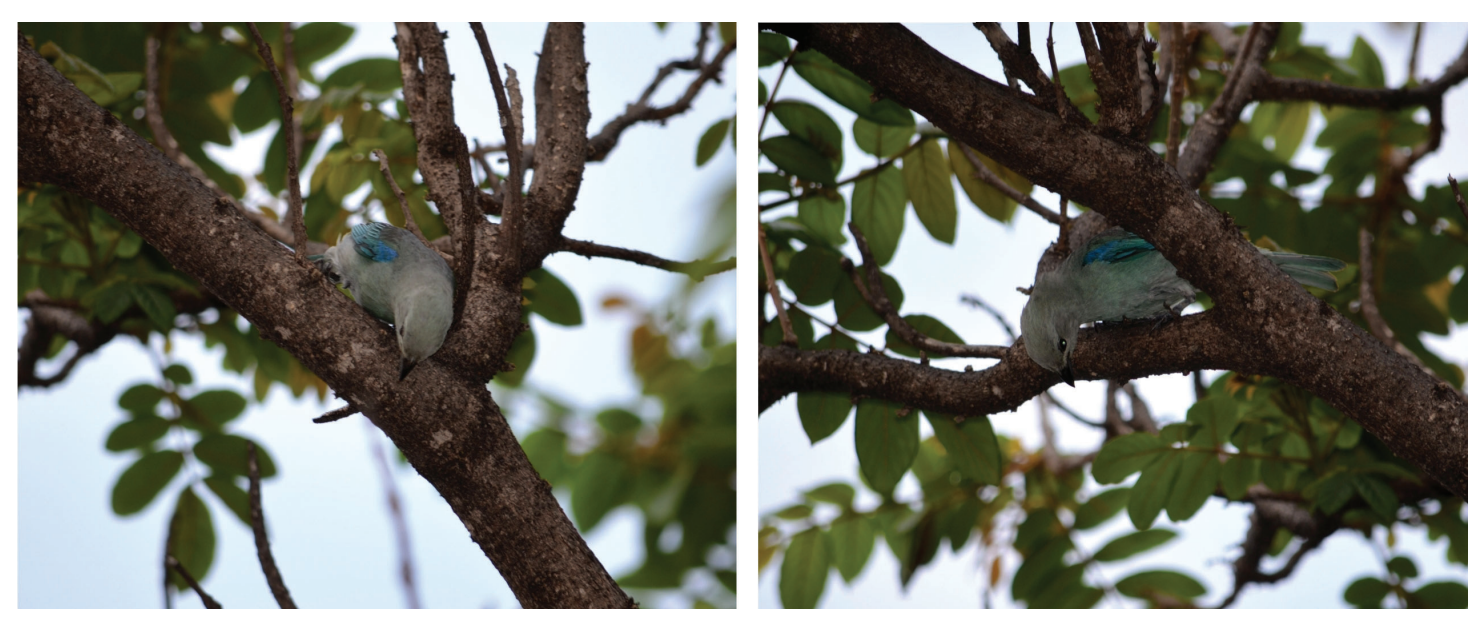

Figura 1. Individuo de tángara azul-gris (T. episcopus) forrajeando en un árbol de tulipán africano, en el zócalo de la ciudad de Chilpancingo, Guerrero. 
2017) y recientemente el sureste del estado de Guerrero (Blancas-Calva et al., 2017). Lo que podría indicar que T. episcopus se ha desplazado hacia áreas modificadas, convirtiéndolas en un hábitat actual de su distribución (Rodríguez-Ruiz et al., 2011).

Este reporte constituye una evidencia más de la capacidad de la especie para vivir en ambientes modificados antropogénicamente. Asimismo, deducimos que individuos de especies de aves de ornato que han escapado accidentalmente de cautiverio, se pueden adaptar a las condiciones propias de los ambientes urbanos estableciendo poblaciones locales, por lo que sugerimos la continuidad de trabajos de ecología urbana que permitan conocer las dinámicas poblacionales de las especies y corroborar el establecimiento de la especie en el sitio.

AGRADECIMIENTOS. A Mario Hernández Maldonado por su apoyo en la determinación de la especie de leguminosa mencionada, y a los revisores anónimos por sus observaciones valiosas para mejorar el escrito.

\section{LITERATURA CITADA}

AOS (American Ornithological Society, Cheklist of North and Middle American Birds). (1998) Available at: http://checklist.aou.org/ (acceseed on October 2017).

Blancas-Calva, E., Blancas-Hernández, J. C., Castro-Torreblanca, M. \& Cano-Nava, G. A. (2017) La tángara azul gris (Thraupis episcopus) en el estado de Guerrero, México. Huitzil, 18(2), 232237.

Clements, J. F. (1991) Birds of the world a check list. Fourth Edition. Ibis, Vista, California. 617 pp.

eBird. (2017) eBird: An online database of bird distribution and abundance. Cornell Lab of Ornithology. Ithaca, New York, EUA Available at: http://ebird.org/ebird/map/ (accessed on October 2017).

Howell, S. N. G., \& Webb, S. (1995) A guide to The Birds of Mexico and Northern Central America. Oxford University Press Inc., New York, E.U.A. 851 pp.

Macaulay Library to Cornell Lab of Ornithology. (2017) Blue-gray Tanager: song. Ithaca, New York, U.S.A. Available at: http://macaulaylibrary.org/search?location_id=\&location_type_id=\&locati on $=\&$ recordist $=\&$ recordist_id $=\&$ catalogs $=\&$ behavior $=\&$ behavior _id=\&tab=audio-list\&taxon_id=12021616\&taxon_rank_id=67\&t axon=blue+gray+tanager (accessed on October 2017).

Monroy-Ojeda, A., \& Grosselet, M. (2008) Registro de la tángara azul gris (Thraupis episcopus) en el estado de Nuevo León, México. Huitzil, 9(2), 29-31.

Navarro, A. G., Peterson, A. T. \& Gordillo-Martínez, A. (2003) Museums working together: the atlas of the birds of Mexico. Pp. 207-225. In: N. Collar, C. Fisher \& C. Feare (Eds.). Why museums matter: avian archives in an age of extinction). Bulletin British Ornithologists' club Supplement 123A. Londres, UK.

Navarro-Sigüenza, A. G. (1998) Distribución geográfica y ecológica de la avifauna del estado de Guerrero, México. Tesis doctoral, Facultad de Ciencias, Universidad Nacional Autónoma de México. México, D. F. 182 p.

Navarro-Sigüenza, A. G., Blancas-Calva, E., Almazán-Núñez, R. C., Hernández-Baños, B. E., García-Trejo, E. A. \& Peterson, A. T. (2016) Diversidad y endemismo de las aves de la Sierra Madre del Sur. Pp. 381-412. In: I. Luna-Vega, D. Espinosa \& R. Contreras-Medina (Eds.). Biodiversidad de la Sierra Madre del Sur. Universidad Nacional Autónoma de México, Ciudad de México.

Navarro-Sigüenza, A. G., García, E., Peterson, A. T. \& Rodríguez-Contreras, V. (2004) Aves. Pp. 391-342. In: A. J. GarcíaMendoza, M. de J. Ordóñez \& M. A. Briones-Salas, (coord.). Biodiversidad de Oaxaca. Instituto de Biología, Universidad Nacional Autónoma de México, Fondo Oaxaqueño para la Conservación de la Naturaleza y World Wildlife Fund. México, D. F.

Navarro-Sigüenza, A. G. \& Peterson, A. T. (2007) Thraupis episcopus (tángara azul gris) residencia permanente. Distribución potencial. Catálogo de metadatos geográficos. Comisión Nacional para el Conocimiento y Uso de la Biodiversidad, México, D.F.

Peterson, R. T. \& Chalif, E. L. (1998) Guía de campo de las Aves de México. Ed. Diana, México, D. F. 473 pp.

Rodríguez-Ruiz, E. R., Garza-Torres, H. A., Ríos-Muñoz, C. A. \& Navarro-Sigüenza, A. G. (2011) La distribución geográfica de la tángara-azul gris (Thraupis episcopus) en hábitats modificados antropogénicamente en México. Revista Mexicana de Biodiversidad, 82, 989-996.

SEMARNAT (Secretaría de Medio Ambiente y Recursos Naturales). (2017) Aprovechamiento de aves canoras y de ornato para fines de subsistencia. Disponible en: www.semarnat.gob.mx/temas/gestion-ambiental/vida-silvestre/aprovechamiento-de-aves-canorasy-de-ornato-para-fines-de (consultado en octubre 2017).

Xeno-canto. (2017) Xeno-canto: sharing bird sounds from around the world. Disponible en: www.xen-canto.org/ (consultado en octubre 2017). 